\title{
The TID model for modulation of large scale electron density models
}

\author{
Reinhart Leitinger and Markus Rieger \\ Institut für Physik, Institutsbereich für Geophysik, Astrophysik und Meteorologie (IGAM), \\ Universität Graz, Austria
}

\begin{abstract}
Various modern applications of empirical electron density models need realistic structures of the electron density distribution with smaller scales than the model background. Travelling Ionospheric Disturbances (TIDs) produce three dimensional and time dependent disturbances of the background ionization. We present a TID model suitable to «modulate» large scale electron density distributions by multiplication. A model TID takes into account the forward tilt of the disturbance wave front, a distinct vertical structure, a fan type horizontal radiation characteristic, geometric dilution and attenuation. More complicated radiation patterns can be constructed by means of superposition. The model TIDs originate from source regions which can be chosen arbitrarily. We show examples for TID modulations of the background model family developed at Trieste and Graz (NeQuick, COSTprof and NeUoG-plas).
\end{abstract}

Key words ionosphere - electron density models Travelling Ionospheric Disturbances - TID model

\section{Introduction}

Travelling Ionospheric Disturbances (TIDs) are the plasma signatures of Atmospheric (acoustic) Gravity Waves (AGWs). They change the plasma distribution of the $F$-layer, which results in fluctuations of electron density as well as of the electron content. The theoretical understanding of these wavelike disturbances was first given by Hines (1960). According to their horizontal wavelengths we distinguish between three classes of TIDs (table I; see e.g., Van Velthoven, 1990).

With these definitions the Small Scale TIDs belong to the «acoustic branch», the others to the «gravity branch». In general, the amplitudes

Mailing address: Dr. Reinhart Leitinger, Institut für Physik, Institutsbereich für Geophysik, Astrophysik und Meteorologie (IGAM), Universität Graz, Universitaetsplatz 5, A8010 Graz, Austria; e-mail: reinhart.leitinger@uni-graz.at of TIDs increase with increasing horizontal wavelengths. Relative peak to peak amplitudes range from 0.001 to a few percent for SSTIDs (which can be considered to be part of «geophysical noise») to $30 \%$ and more for LSTIDs. There are two source regions for the AGWs behind the TIDs: 1) the lower atmosphere (troposphere and lower stratosphere) where it is thought that AGWs behind most of the MSTIDs are generated; 2) the lower thermosphere in the auroral oval (E-region heights) where it is thought that most of the LSTIDs (and some of the MSTIDs) are generated (see $e . g$., Leitinger, 1992; Kirchengast, 1996). In mid-latitudes most LSTIDs propagate equatorwards, those which travel polewards have smaller amplitudes and are thought to have their source regions in the other hemisphere. In some cases the occurrence of large scale TIDs could be linked to auroral substorm activity (Kirchengast, 1997; see also Kishcha et al., 1993).

The main purpose of the TID model presented here is the combination by multiplication with a suitable electron density background model like the «family» of models developed at Trieste 
Table I. TID classes according to their horizontal scales.

\begin{tabular}{cccc}
\hline \hline Class & Horizontal wavelength & Periods & Horizontal phase velocities \\
\hline Large Scale (LSTIDs) & $>1000 \mathrm{~km}$ & $(0.5 \ldots 3) \mathrm{h}$ & $(300 \ldots 1000) \mathrm{m} / \mathrm{s}$ \\
Medium Scale (MSTIDs) & $(100 \ldots 1000) \mathrm{km}$ & 12 min $\ldots 1 \mathrm{~h}$ & $(100 \ldots 300) \mathrm{m} / \mathrm{s}$ \\
Small Scale (SSTIDs) & $<100 \mathrm{~km}$ & A few minutes & $<200 \mathrm{~m} / \mathrm{s}$ \\
\hline
\end{tabular}

and Graz (NeQuick, COSTprof and NeUoGplas) or the International Reference Ionosphere (IRI). In this way we are able to demonstrate the influence of TIDs on application systems like satellite assisted positioning as used in surveying or on the use of polar orbiting satellite beacons as novel ionospheric data sources (high resolution ionosphere tomography; see e.g., Leitinger, 1999) or on Radio Occultation data (see e.g., Jakowski et al., 2004).

\subsection{Atmospheric Gravity Waves (AGWs) - A very short overview}

Consider a stable homosphere with a given temperature profile $T=T(z)$ ( $z$ : height). With knowledge of the acceleration of gravity $g=g(z)$ and with one pressure and mass density value we also know the pressure profile $p=p(z)$ and the mass density profile $\rho=\rho(z)$. Starting with displacing air parcels adiabatically in the vertical by $\Delta z$ one can show that the buoyancy force acts as a restoring force (see Van Velthoven, 1990, and references therein; Prölss, 2001)

$$
\rho_{0} \frac{d^{2}(\Delta z)}{d t^{2}}=g\left(\frac{d \rho_{0}}{d z}+\frac{\rho_{0} g}{c_{s}^{2}}\right) \Delta z
$$

$c_{s}=\sqrt{\gamma\left(p_{0} / \rho_{0}\right)}$ is the velocity of sound, $\gamma=c_{p} / c_{v}$ is the ratio of specific heats at constant pressure and at constant volume, $p_{0}$ and $\rho_{0}$ are pressure and mass density at the starting level for the displacement. This equation (Newton's second law) can be considered as a differential equation for an oscillation with the general solution (for small amplitudes $A) \Delta z=A \exp \left(j \omega_{B} t\right) ; j=\sqrt{-1}, \omega_{B}^{2}=$ $=\omega_{b}^{2}+\left(g / c_{s}^{2}\right)\left(d\left(c_{s}^{2}\right) / d z\right)$ is the square of the (general) Brunt-Väisälä or buoyancy angular frequency, $\omega_{b}^{2}=\left[(\gamma-1) g^{2}\right] / c_{s}^{2}$ being the square of the isothermal Brunt-Väisälä angular frequency.
Forcing of oscillations will lead to wave propagation because the air parcel in consideration is connected to its surroundings.

Deriving wave solutions from the linearized hydrodynamic equations for an isothermal atmosphere over a flat Earth leads to the following dispersion relation for AGWs, first published by Hines (1960)

$$
k_{z}^{2}=\frac{\omega_{b}^{2}-\omega^{2}}{\omega^{2}} k_{x}^{2}-\frac{\omega_{a}^{2}-\omega^{2}}{c_{s}^{2}}
$$

with the «acoustic cut-off angular frequency» $\omega_{a}=(\gamma g) /\left(2 c_{s}\right) . k_{z}$ and $k_{x}$ are the vertical and horizontal components of the wave vector $\boldsymbol{k}$.

The waves described by this relation can be categorised as follows (see e.g., Van Velthoven, 1990):

a) $\omega>\omega_{a}$, acoustic branch; if $\omega>>\omega_{a}$ the dispersion relation degenerates to the dispersion relation for pure acoustic waves $k^{2}=\omega^{2} / c_{s}^{2}$;

b) $\omega_{b}<\omega<\omega_{a}$, evanescent waves; wave equation becomes diffusion equation;

c) $\omega<\omega_{b}$, gravity wave branch; if $\omega<<\omega_{b}$ the dispersion relation degenerates to the dispersion relation for pure gravity waves $k_{z}^{2}=\left(\omega_{b}^{2} / \omega^{2}\right)$. $\cdot k_{x}^{2}-\left(\omega_{a}^{2} / c_{s}^{2}\right)$

\subsection{The response of the ionosphere to $A G W s$}

The equation of continuity for electrons can be written as

$$
\frac{\partial N_{e}}{\partial t}=q_{e}-L_{e}-\nabla \cdot\left(N_{e} \boldsymbol{v}_{e}\right)
$$

where $N_{e}$ is the electron density; $\boldsymbol{v}_{e}$, the electron velocity; $q_{e}$, the electron production rate; and $L_{e}$, the loss of electrons by recombination.

The electron density can be split into a stationary background electron density $\left(N_{e 0}\right)$ and a perturbation $\left(N_{e 1}\right)$, due to the passage of the 
AGW. Assuming $\boldsymbol{v}_{e 1}$ to be the velocity induced by the AGW leads to

$$
N_{e}=N_{e 0}+N_{e 1}, \boldsymbol{v}_{e}=\boldsymbol{v}_{e 0}+\boldsymbol{v}_{e 1} .
$$

Assuming no perturbation in production and loss and setting $\boldsymbol{v}_{e 0}=0$ gives

$$
\frac{\partial N_{e 1}}{\partial t}=-\nabla \cdot\left(N_{e 0} \boldsymbol{v}_{e 1}\right) \text {. }
$$

The relation between $\boldsymbol{v}_{e 1}$ and $\boldsymbol{v}_{n 1}$ is given by a balance between the Lorentz force and the «ion drag» force

$$
e\left(\boldsymbol{v}_{e 1} \times \boldsymbol{B}\right)+m_{i} v\left(\boldsymbol{v}_{e 1}-\boldsymbol{v}_{n 1}\right)=0
$$

where $\boldsymbol{v}_{n 1}$ is the perturbation velocity of the neutral gas; $\boldsymbol{B}$, the geomagnetic induction vector; $m_{i}$, the ion mass; and $v_{i}$, the effective ionneutrals collision frequency.

In the $F$-region of the ionosphere $(h>150$ $\mathrm{km}$ ) the mobility of the ions perpendicular to the geomagnetic field vector is negligibly small and we can approximate

$$
\boldsymbol{v}_{e 1} \approx\left(\boldsymbol{v}_{n 1} \cdot \boldsymbol{b}\right) \boldsymbol{b}
$$

where $\boldsymbol{b}$ is the unit vector in the direction of the geomagnetic field.

This leads to the electron density disturbance in the form

$$
N_{e 1} \doteq\left(\boldsymbol{v}_{n} \cdot \boldsymbol{b}\right)\{j(\boldsymbol{b} \cdot \boldsymbol{k})-j(\boldsymbol{b} \cdot \nabla)\} N_{e 0} .
$$

If in (1.7) the second term of the expression in \{\} can be neglected we approximate

$$
\begin{aligned}
& \frac{N_{e 1}}{N_{e 0}} \doteq \frac{k\left(\boldsymbol{v}_{n} \cdot \boldsymbol{b}\right)}{\omega} \cos (\boldsymbol{\Xi})=\frac{U_{n}}{v_{p h}} \cos (\Sigma) \cos (\boldsymbol{\Xi}) \\
& \cdot \cos \left(k_{x} s+k_{z} h+\Psi(h)-\omega t\right)
\end{aligned}
$$

where $U_{n}$ is the amplitude of the AGW; $v_{p h}$, the phase velocity of the AGW; $s$, the horizontal coordinate; $h$, the height coordinate; and $\Psi(h)$ is a height dependent phase constant.

The projection factors are $\cos (\boldsymbol{\Xi})=(\boldsymbol{k} \cdot \boldsymbol{b}) /|\boldsymbol{k}|$ and $\cos (\Sigma)=\left(\boldsymbol{v}_{n 1} \cdot \boldsymbol{b}\right) /\left|\boldsymbol{v}_{n 1}\right|$.

Energy transport upwards corresponds to a downward directed wave normal, $k_{z}<0$. Then $\chi=\operatorname{atan}\left(-k_{x} / k_{z}\right)$ is the famous «forward tilt» of the planes of constant phase of the AGWs. For AGWs $\Psi$ is a constant value. Because of the projection unto the geomagnetic field it is (slightly) height dependent in the TID case (compare e.g., Francis, 1975; Morgan and Calderón, 1978).

The approximation (1.6) implies, that there is no ionospheric response to AGWs with wave vectors perpendicular to the magnetic field. Below the $F$-region the condition (1.6) is not valid and so variations of $\boldsymbol{v}_{n}$ perpendicular to the geomagnetic field cannot be excluded.

\subsection{AGW signatures in Total Electron Content (TEC)}

The integration of electron density along slant rays from a satellite transmitter to a ground receiver gives (slant) Total Electron Content. AGW signatures are partly smoothed out. Perpendicular to the geomagnetic field vector we see no TIDs at all. It is necessary to have a geomagnetically meridional component to observe TIDs in TEC, but even with this geometry we have a distinct «aspect sensitivity» (Georges and Hooke, 1970).

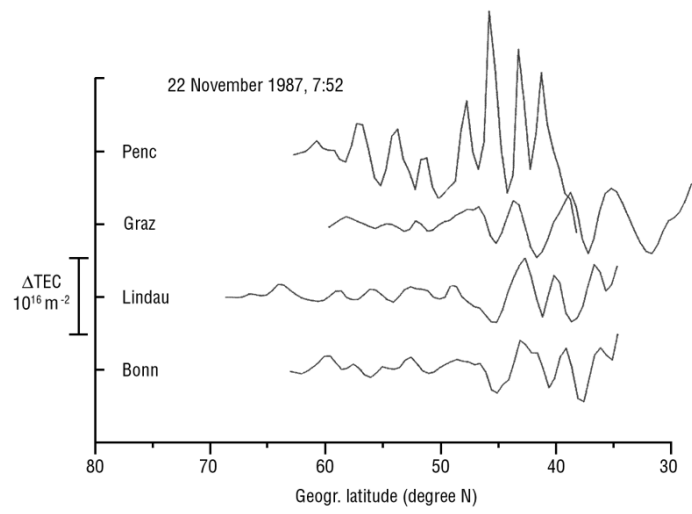

Fig. 1. Examples for TIDs in observed electron content (Leitinger, 1992). NNSS Difference Doppler observations, band pass filtered data. Receiving stations: Bonn $\left(50.5^{\circ} \mathrm{N}, 6.9^{\circ} \mathrm{E}\right)$, Lindau/Harz $\left(51.6^{\circ} \mathrm{N}, 10.1^{\circ} \mathrm{E}\right)$, Graz $\left(47.1^{\circ} \mathrm{N}, 15.5^{\circ} \mathrm{E}\right)$, Penc $\left(47.8^{\circ} \mathrm{N}, 19.3^{\circ} \mathrm{E}\right)$. Marked amplitude range: $1 \times 10^{16} \mathrm{~m}^{-2}$. Note the differences in TID amplitudes for the closely spaced receiving stations. For more examples see Putz et al. (1990), Leitinger and Putz (1991). 

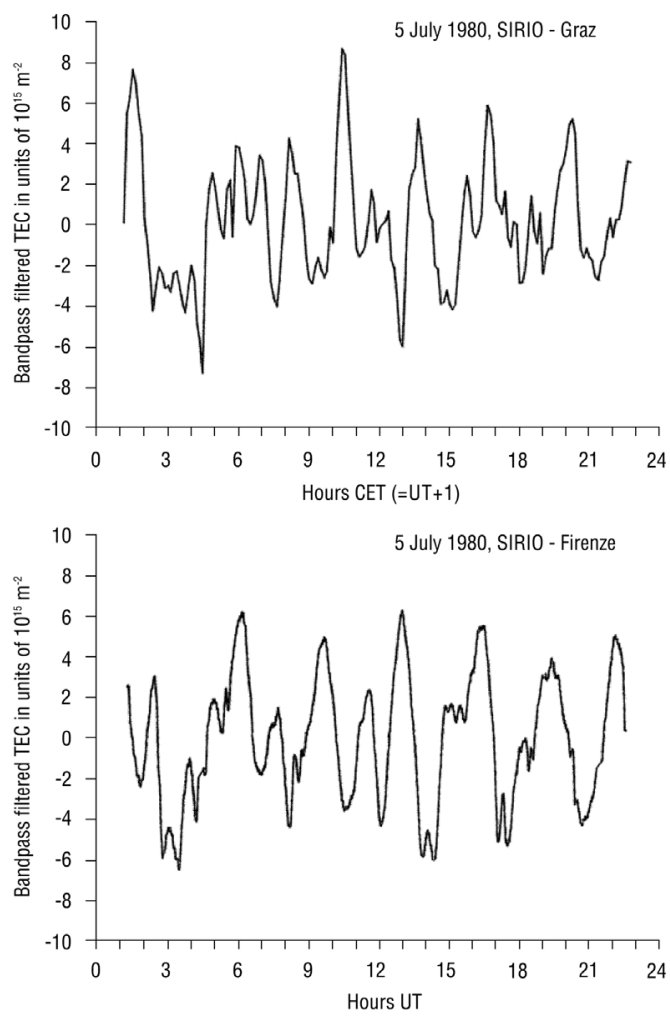

Fig. 2. Examples for TIDs in observed electron content (Leitinger, 1992). SIRIO Faraday observations, band pass filtered data. Receiving stations: Graz $\left(47.1^{\circ} \mathrm{N}, 15.5^{\circ} \mathrm{E}\right)$ and Firenze $\left(43.0^{\circ} \mathrm{N}, 10.7^{\circ} \mathrm{E}\right) . \mathrm{Ab}-$ scissae in hours CET (top) or UT (bottom), ordinates in units of $10^{15} \mathrm{~m}^{-2}$. For more examples see Putz et al. (1992).

Because of the tilt of AGW fronts the TID disturbance in TEC is smoothed out polewards of the receiving station if the AGW propagates equatorwards. The tilt of the wavefront itself does not appear (figs. 1 and 2: observed TIDs; figs. 5 to 7 : model TIDs). Projection from slant to vertical in $\mathrm{a}$ «mean ionospheric height» does not change this situatuation.

\section{Some TID observations}

Travelling ionospheric disturbances have been observed with many different instruments.
For TIDs in GPS data see Rieger and Leitinger (2002). Here we show two examples for TIDs observed by means of propagation effects on satellite signals. Figure 1 shows TIDs observed by means of the Difference Doppler effects on signals of polar orbiting satellites. The evaluation of the observations gave the latitude dependence of the vertical electron content (TEC) of the ionosphere. The disturbance by large scale TIDs is demonstrated by means of band pass filtering of the electron content data. Figure 1 is a clear example for the effect of the tilt of the wave fronts and of the filtering effect introduced by the geomagnetic field. The equatorward travelling TIDs appear only equatorwards of the receiving stations. Furthermore the data for the closely spaced receiving stations show strong differences in TID amplitudes which indicates that the AGWs behind the Travelling Ionospheric Disturbances are not radiated from an isotropic source but have a distinct «radiation pattern».

The second example (fig. 2) shows TIDs in Faraday effect observations on the VHF signal

Table II. Examples for the large scale «model basis».

The «family» of models developed at Trieste and Graz

$$
\begin{gathered}
\text { NeQuick }(Q), \\
\text { COSTprof }(C) \\
\text { and } \\
\text { NeUoG-plas }(P)
\end{gathered}
$$

are profilers using the peaks of the $E$-layer, the $F 1$ layer and the F2-layer as anchor points. They are identical from $100 \mathrm{~km}$ to the $F 2$ peak (Epstein layer formulations).

$C$ and $P$ have identical ionosphere topsides (height aligned $\mathrm{O}^{+}-\mathrm{H}^{+}$Diffusive Equilibrium - DE), $Q$ uses a semi-Epstein layer with a height dependent thickness parameter.

Above $2000 \mathrm{~km} P$ uses a magnetic field aligned $\mathrm{H}^{+}$ DE («plasmasphere»).

The models allow

- to be updated with actual data from various sources;

- to be adjusted to disturbed conditions;

- to be combined with smaller scale models;

- to calculate a variety of propagation effects along arbitrarily chosen raypaths.

Average ratio of calculation times $Q: C: P=1: 2: 4$. 


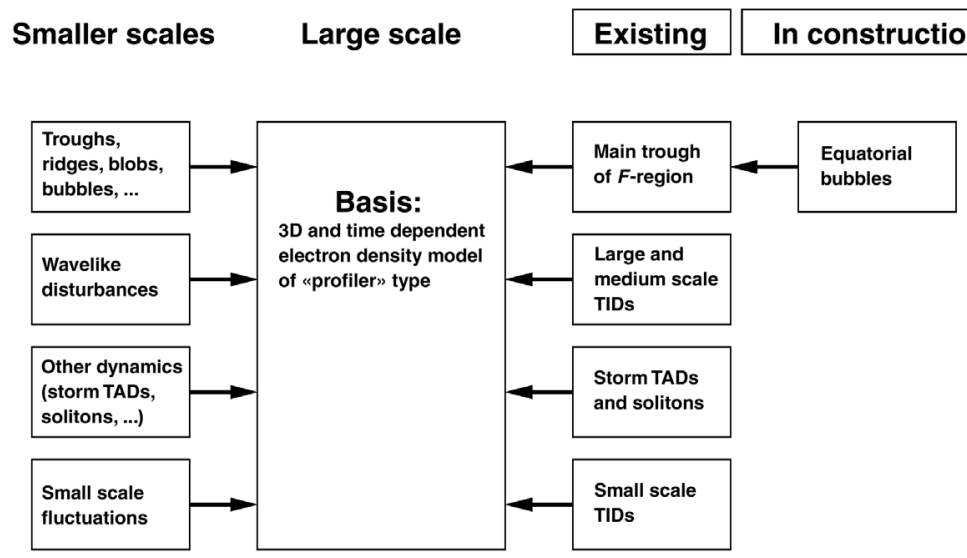

Fig. 3. Scheme for the multiplicative «modulation» of electron density models to add smaller scale structures.

of the geostationary satellite SIRIO. In this case evaluation of the raw data gives the time dependence of vertical electron content. Band pass filtering reveals LSTIDs of comparatively high amplitudes. Surprisingly there is not much amplitude variation over the day. This means that the relative TID amplitudes have been larger during nighttime than during daytime. This is in accordance with the idea that AGW attenuation is caused by «ion drag» and therefore stronger during daytime (higher ionization) than during nighttime.

\section{Model requirements and «modulation» technique}

In general, three dimensional and time dependent electron density models for the ionosphere of the Earth, like the «family» of models developed at Trieste and Graz, NeQuick, COSTprof, NeUoG-plas (table II) (Hochegger et al., 2000) or the International Reference Ionosphere (IRI; Bilitza, 2001) provide the large scale «background» only. If the models give electron density for fixed universal time as a function of geographic coordinates latitude, longitude and height, smaller scale structures can be added by a multiplicative «modulation» technique (fig. 3) (Leitinger et al., 2002)

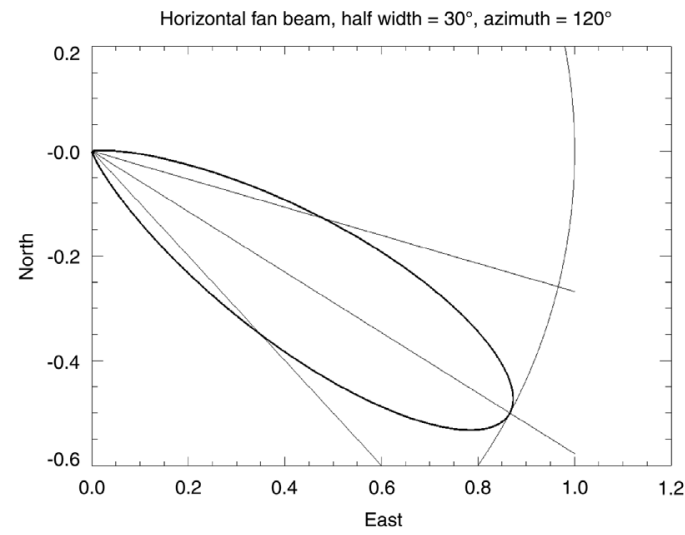

Fig. 4. Horizontal radiation pattern of the TID model: radiation into a fan beam of given half width and azimuth.

$$
\begin{aligned}
& \boldsymbol{M}(h, \varphi, \lambda, t)=\boldsymbol{L}(h, \varphi, \lambda, t) T(h, \varphi, \lambda, t) \\
& \cdot\left[S_{1}(h, \varphi, \lambda, t)+\cdots+S_{n}(h, \varphi, \lambda, t)\right]
\end{aligned}
$$

where $\boldsymbol{M}$ is the resulting electron density model; $\boldsymbol{L}$, the large scale model (member of our model family, IRI, etc.); $\boldsymbol{T}$ and $\boldsymbol{S}$, the modulations $e . g$., for the main trough and for TIDs; $h$, the height; $\varphi$, geographic latitude; $\lambda$, the geographic longitude; and $t$, the universal time. 


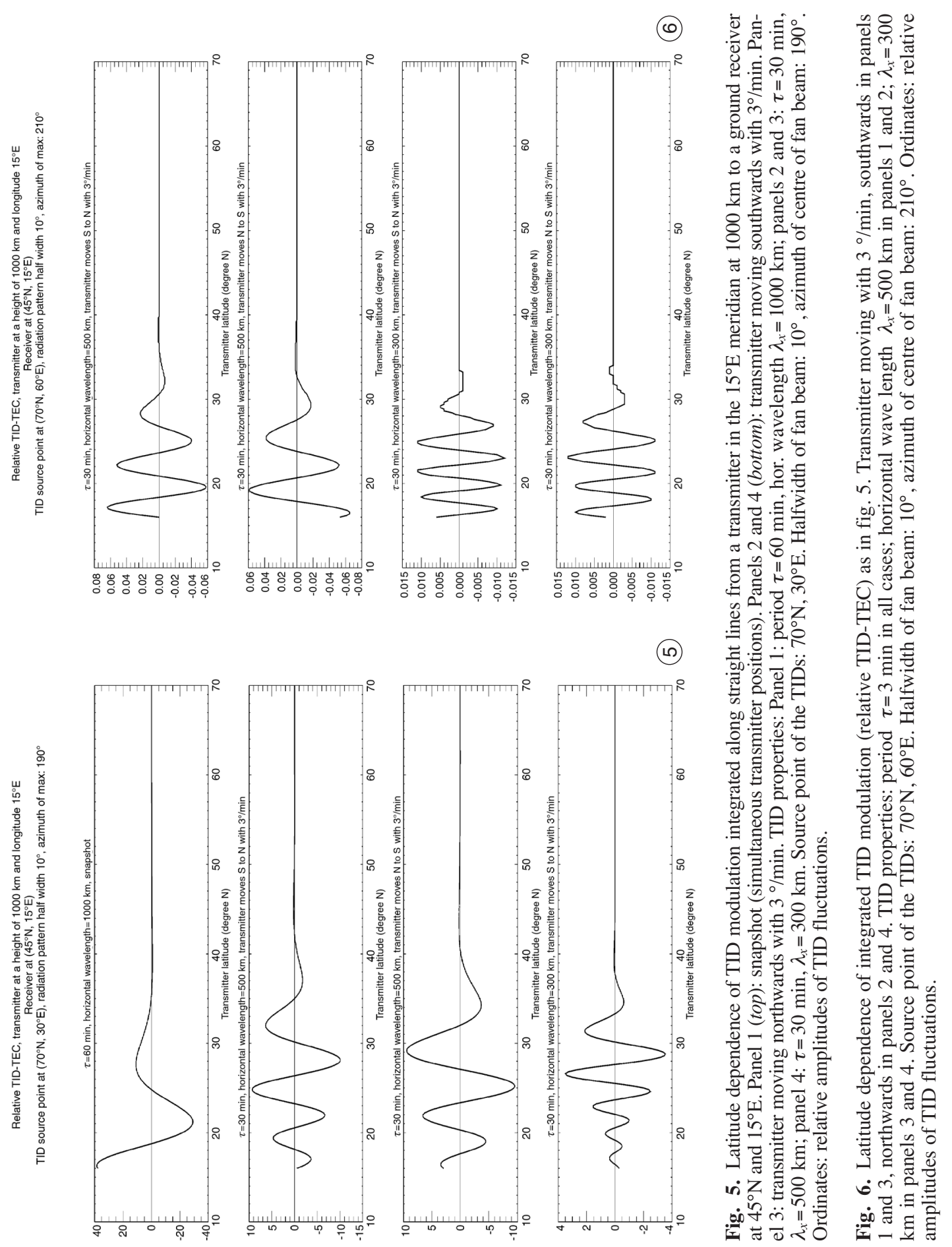




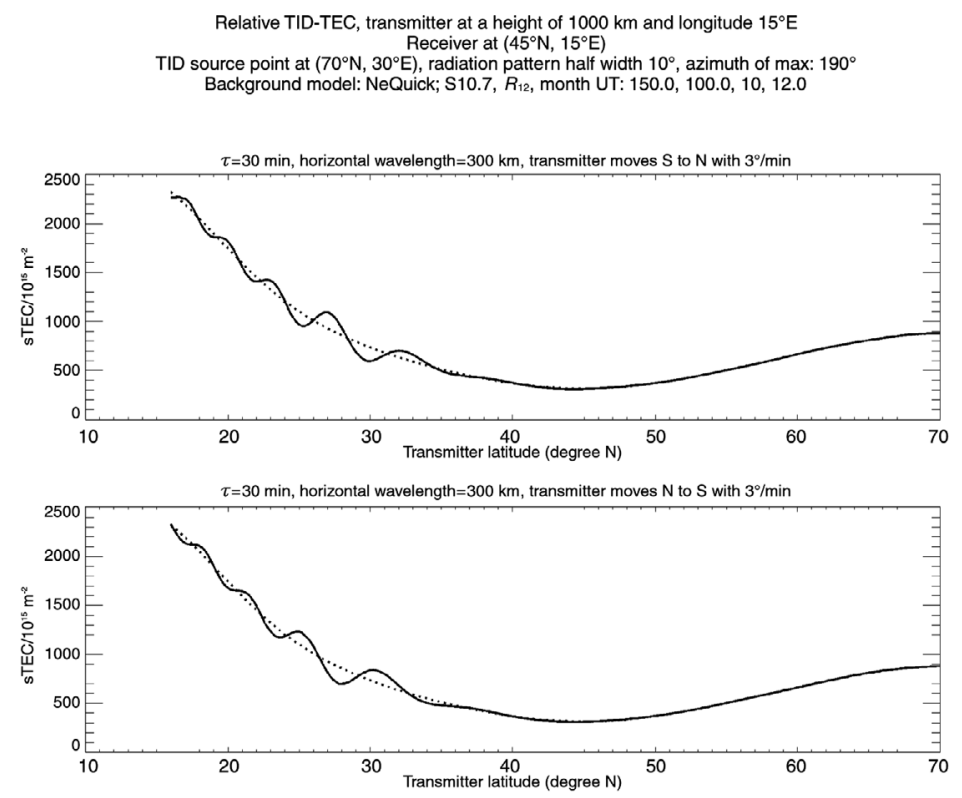

Fig. 7. Latitude dependence of TID modulated electron density model NeQuick. Geometry and TID properties as in fig. 5, panels 3 and 4. Solid lines: TID modulated electron density. Dotted lines: background model.

\section{The TID model}

The TID model makes use of the following properties of Atmospheric Gravity Waves (AGWs):

- the horizontal component $k_{x}$ of the wave vector;

- the AGW period $\tau=2 \pi \omega$;

- the dispersion relation to derive the vertical component $k_{z}$ of the wave vector;

- the velocity of the disturbance $\boldsymbol{v}_{n}=\boldsymbol{U}_{n} \cos$. . $\left(k_{x} s+k_{z} h+\Psi(h)-\omega t\right)$ (where $s$ is the horizontal coordinate; $h$, theheight; $t$, the time; and $\Psi$ is a height dependent phase constant).

Derived quantities are among others the horizontal and vertical wave lengths and phase velocities $\lambda_{x}=2 \pi / k_{x}, \lambda_{z}=2 \pi / k_{z}, v_{x}=\omega / k_{x}$, $v_{z}=\omega / k_{z}$.

The model takes into account the projection factors discussed above, geometric dilution, horizontal and vertical attenuation. Since the radiation patterns are far from isotropic the model assumes radiation into «fan beams» (fig. 4) and allows the combination of several beams originating in one or in several different source «points». Presently we are using vertical «source lines» instead of real «source points», meaning that the vertical structure does not change with propagation. Because of a modification of the «near field» formulation it is not necessary to exclude the source points.

Some details:

- The TIDs originate in chosen «source points».

- A TID travels in a «fan beam» defined by its azimuth and its half width; the wave properties are given by the wave period $\tau$ and by the horizontal wave length $\lambda_{x}$, the vertical wave length follows from the dispersion relation.

- For the vertical structure a Chapman profile was adopted defined by a scale height $H_{T}$ and by a peak height $h_{m T}$. Values chosen for the examples shown: $H_{T}=100 \mathrm{~km}, h_{m T}=250 \mathrm{~km}$.

- The forward tilt of the wave fronts is produced by $\left(-k_{x} / k_{z}\right)$ in accordance with the dispersion relation of the AGWs.

- The geometric dilution of horizontally travelling AGWs and horizontal attenuation are also taken into account. 


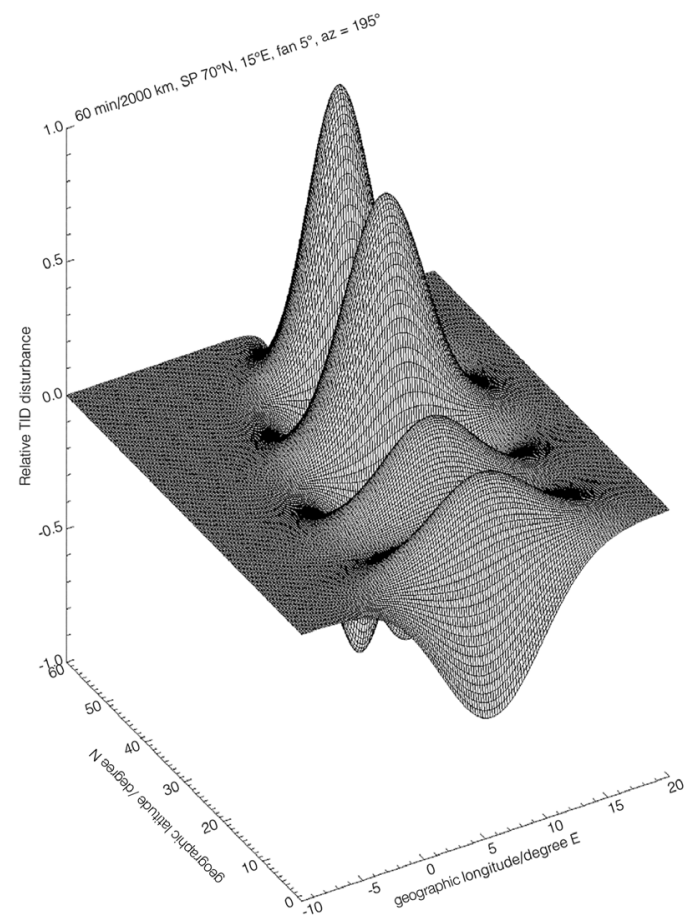

Fig. 8. Example from the TID model: TID in a fixed height of $250 \mathrm{~km}$. Horizontal coordinates: geographic longitude $\left(-10^{\circ} \mathrm{E}-20^{\circ} \mathrm{E}\right)$ versus geographic latitude $\left(0^{\circ} \mathrm{N}\right.$ to $\left.60^{\circ} \mathrm{N}\right)$. TID properties: wave period $60 \mathrm{~min}$, horizontal wavelength $2000 \mathrm{~km}$, source point in $70^{\circ} \mathrm{N}, 15^{\circ} \mathrm{E}$, half width of fan beam: $5^{\circ}$, azimuth of centre of fan beam: $195^{\circ}$.

- The model allows superposition of several TID wave trains. An example for a single TID wave train is given in figs. 8 and 9 .

\section{Discussion and conclusions}

The main purpose of our TID model is to provide a «modulation» for large scale electron density models used primarily for the numerical calculation of transionospheric propagation parameters, one of the most important being satellite to ground electron content. The TIDs have a simplified vertical structure (Chapman layer) and therefore are not well suited for $E$-region and lower $F$-region applications. Comparisons of observations with model results show that we obtain realistic TID signatures in electron content (see figs. 1 and 5 to 7).

The TID model should not be used for tests of AGW sources and propagation paths. One should not expect realistic «near field» results.

The TID formulation has been used successfully to model interfering smaller scale TIDs as observed by means of a dense network of dual frequency GPS receivers (Rieger and Leitinger, 2005).

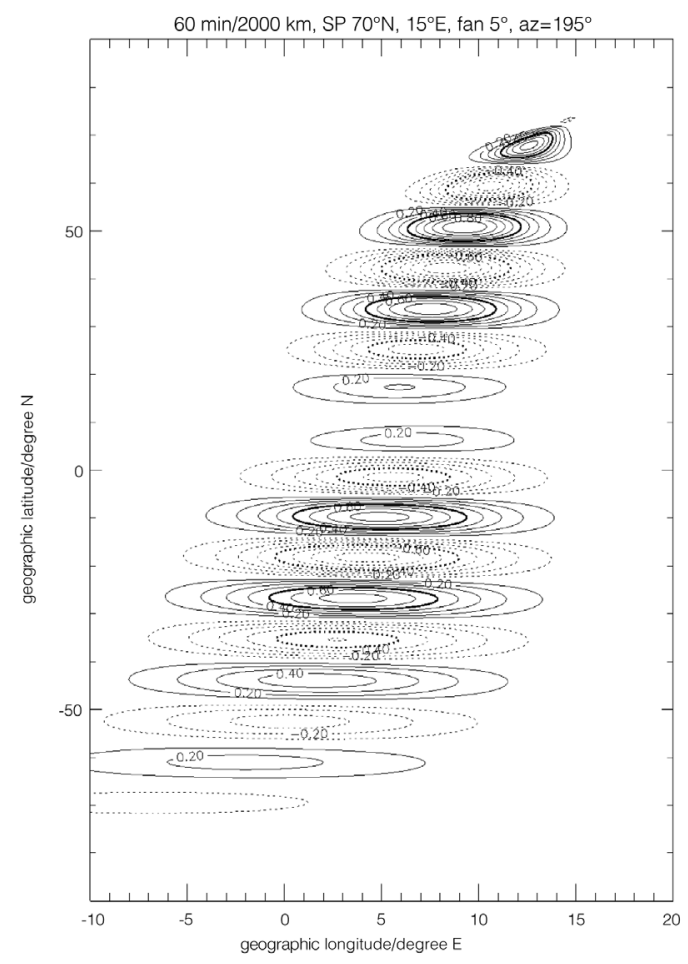

Fig. 9. Example from the TID model: TID in a fixed height of $250 \mathrm{~km}$. Isolines of relative TID amplitude over a geographic coordinate system (longitude $-10^{\circ} \mathrm{E} \ldots 20^{\circ} \mathrm{E}$ versus latitude $-90^{\circ} \mathrm{N} \ldots 90^{\circ} \mathrm{N}$ ). Solid lines: positive levels $(0.1,0.2, \ldots, 0.9)$, dotted lines: negative levels $(-0.1,-0.2, \ldots-0.9)$. TID properties: wave period $60 \mathrm{~min}$, horizontal wavelength $2000 \mathrm{~km}$, source point in $70^{\circ} \mathrm{N}, 15^{\circ} \mathrm{E}$, half width of fan beam: $5^{\circ}$, azimuth of centre of fan beam: $195^{\circ}$. The «projection effect» is cleanly seen in the latitude region around $10^{\circ} \mathrm{N}$ (AGW wind nearly perpendicular to geomagnetic induction vector). 


\section{REFERENCES}

BilitZA, D. (2001): International Reference Ionosphere 2000, Radio Sci., 36, 261-275.

FRANCIS, S.H. (1975): Global propagation of atmospheric gravity waves: a review, J. Atmos. Terr. Phys., 37, 10111054.

Georges, T.M. and W.H. Hooke (1970): Wave-induced fluctuations in the ionospheric electron content: a model indicating some observational biases, J. Geophys. Res., 75, 6295-6308.

HinEs, C.O. (1960): Internal atmospheric gravity waves at ionospheric heights, Canad. J. Phys., 38, 1441-1481.

HochegGER, G., B. NAVA, S.M. RADICELLA and R. LEITINGER (2000): A family of ionospheric models for different uses, Phys. Chem. Earth (C), 25, 307-310.

JAKOWSKI, N., R. LEITINGER and M. ANGLING (2004): Radio occultation techniques for probing the ionosphere, Ann. Geophysics, 47 (suppl. to no.2/3), 1049-1066.

KIRCHENGAST, G. (1996): Elucidation of the physics of the gravity wave - TID relationship with the aid of theoretical simulations, J. Geophys. Res., 101, 13353-13368.

KIRCHENGAST, G. (1997): Characteristics of high-latitude TIDs from different causative mechanisms deduced by theoretical modelling, J. Geophys. Res., 102, 45974612.

Kishcha, P.V., V.M. SHASHUnKina and E.E. GonChaRova (1993): Updating the IRI ionospheric model for effects of substorms, Adv. Space Res., 13, 67-70.

LEITINGER, R. (1992): Travelling Ionospheric Disturbances (TIDs) - Wissensstand und neuere Entwicklungen. Kleinheubacher Berichte, 35, 1-14.

LEITINGER, R. (1999): Ionospheric tomography, in Review of Radio Science, 1996-1999, edited by W.R. STONE (Oxford University Press, Oxford, U.K.), 581-623.
LEITINGER, R. and E. PUTZ (1991): Untersuchung der Begrenzung von F-Schicht-Prognosen durch den Einfluß atmosphärischer Schwerewellen, Kleinheubacher Berichte, 34, 535-542.

Leitinger, R., S.M. RADicElla and B. NAVA (2002): Electron density models for assessment studies - new developments, Acta Geod. Geophys. Hung., 37, 183-193.

Morgan, M.G. and C.H.J. CAlderón (1978): Testing Hooke's perturbation formula for the production of TID's by gravity waves, J. Geophys. Res., 83, 57375740 .

Prölss, G.W. (2001): Physik des erdnahen Weltraums: Eine Einführung (Springer, Berlin).

Putz, E., R. Leitinger, G.K. Hartmann, and P. Bencze (1990): Simultane Beobachtung von TID's an mehreren Beobachtungsstationen, Kleinheubacher Berichte, 33, 113-119.

Putz, E., R. Leitinger, N. Jakowski, and P. Spalla (1992): Untersuchung zur Interpretation von SchwerewellenSignaturen in der Kombination von Elektroneninhaltsdaten aus verschiedenen Quellen, Kleinheubacher Berichte, 35, 105-112.

Rieger, M. and R. LeItinger (2002): Assessment of TID activity from GPS phase data collected in a dense network of GPS receivers, Acta Geod. Geophys. Hung., 37, 327-341.

RIEGER, M. and R. LEITINGER (2005): Simulation studies for the retrieval of smaller scale electron content structures from regional GPS observations, in Proceedings of the International Beacon Satellite Symposium 2004 (in press).

VAN Velthoven, P.F.J (1990): Medium scale irregularities in the ionospheric electron content, Ph.D. Thesis (Technische Universiteit Eindhoven, NL-5600 MB Eindhoven). 